\title{
PAPEL DEL EMPRENDIMIENTO EN EL CONFLICTO Y POSTCONFLICTO: UNA REVISIÓN SISTEMÁTICA DE LITERATURA
}

\section{ROLE OF ENTREPRENEURSHIP IN THE CONFLICT AND POST-CONFLICT: A SYSTEMATIC REVIEW OF LITERATURE}

\section{PAPEL DO EMPREENDEDORISMO NO CONFLITO E PÓS-CONFLITO: UMA REVISÃO SISTEMÁTICA DA LITERATURA}

Claudia Magali Solarte Solarte; Martha Lida Solarte Solarte; Gloria Alicia Rivera Vallejo

Magíster en mercadeo, Universidad de Manizales, Colombia. Docente Programa de Administración de Empresas, Universidad Cesmag. ORCiD: 0000-0001-8844-2070. E-mail: cmsolarte@unicesmag.edu.co, Colombia.

Magíster en mercadeo, Universidad de Manizales, Colombia. Docente Programa de Administración Financiera, Corporación Universitaria Minuto de Dios, Uniminuto. ORCiD: 0000-0003-3348-1368. E-mail: msolarteso@ uniminuto.edu.co, Colombia.

Magíster en educación desde la diversidad, Universidad de Manizales, Colombia. Docente Programa de Administración de Empresas, Universidad Cesmag. ORCiD: 0000-0001-7568-7466. E-mail: garivera@unicesmag.edu.co, Colombia.

Recibido: 14 de agosto de 2020

Aprobado: 12 de noviembre de 2020

DOI: https://doi.org/10.22267/rtend.212201.161

\section{Resumen}

El artículo tiene como objetivo examinar el emprendimiento en Colombia como una estrategia de inclusión y productividad con víctimas y excombatientes en proceso de reincorporación a la sociedad. 
Como metodología, se realizó una revisión de publicaciones científicas en las bases de datos Scopus, Scielo, Redalyc, Science Direct y Google Scholar, acogiendo artículos que incluyeran emprendimiento, conflicto, postconflicto y reincorporación, en un periodo de 2008 a 2019. Los resultados ofrecieron información respecto al continente de ubicación, donde la mayoría son de Colombia, los enfoques de investigación utilizados y las estrategias de emprendimiento propuestas para procesos de reintegración. Como conclusión, si bien el emprendimiento constituye una estrategia válida de inclusión y productividad para la reinserción a la sociedad, esto necesita de un proceso educativo para que los implicados se apropien de prácticas y conceptos propios para asumir la creación de empresas y proyectos productivos.

Palabras clave: conflicto; creación de empresas; desarrollo económico; emprendimiento; políticas públicas.

JEL: D74; J18; L26; M13; R11

\begin{abstract}
The article presents a systematic review of documents related to entrepreneurship in conflict and postconflict periods in different countries. The objective is to examine entrepreneurship as a strategy of inclusion and productivity with victims and former combatants in the process of reincorporation into society. As a methodology, a review of scientific publications was carried out in the Scopus, Scielo, Redalyc, Science Direct and Google Scholar databases, hosting articles that included entrepreneurship, conflict, post-conflict and reintegration, in a period from 2008 to 2019. It was taken into It counts as an inclusion criterion that they refer to internal conflicts of the countries and not to wars and agreements between States. The results offer information regarding the location of the articles on conflict and post-conflict, the research approaches used and the proposed entrepreneurship strategies for reintegration processes. In conclusion, although entrepreneurship constitutes a valid strategy of inclusion and productivity for reintegration into society, this requires an educational process so that those involved take ownership of their own practices and concepts to assume the creation of companies and productive projects. they must have institutional support at the national and local level to achieve successful experiences.
\end{abstract}

Keywords: conflict; business creation; economic development; entrepreneurship; public politics. JEL: D74; J18; L26; M13; R11 


\section{Resumo}

O artigo apresenta uma revisão sistemática de documentos relacionados ao empreendedorismo em períodos de conflito e pós-conflito em diferentes países. O objetivo é examinar o empreendedoris mo como estratégia de inclusão e produtividade com vítimas e ex-combatentes em processo de reinserção na sociedade. Como metodologia, foi realizada uma revisão das publicações científicas nas bases de dados Scopus, Scielo, Redalyc, Science Direct e Google Scholar, hospedando artigos que incluíam empreendedorismo, conflito, pós-conflito e reintegração, no período de 2008 a 2019. Foi realizado Conta como critério de inclusão que se refiram a conflitos internos dos países e não a guerras e acordos entre Estados. Os resultados oferecem informações sobre a localização dos artigos sobre conflito e pós-conflito, as abordagens de pesquisa utilizadas e as estratégias de empreendedorismo propostas para os processos de reintegração. Em conclusão, embora o empreendedorismo constitua uma estratégia válida de inclusão e produtividade para a reinserção na sociedade, isso requer um processo educativo para que os envolvidos se apropriem de suas próprias práticas e conceitos para assumir a criação de empresas e projetos produtivos. eles devem ter apoio institucional em nível nacional e local para alcançar experiências bem-sucedidas.

Palavras-chave: conflito; criação de negócios; desenvolvimento económico; empreendedorismo; políticas públicas.

JEL: D74; J18; L26; M13; R11

\section{Introducción}

El Acuerdo de paz en Colombia (2016), firmado por el Gobierno y el grupo insurgente Fuerzas Armadas Revolucionarias de Colombia (FARC), generó expectativas en todos los ámbitos de la sociedad, desde el sector del campesinado que aspira a una recomposición del campo, sus formas de propiedad y de trabajo hasta una apertura democrática con participación política de todos los sectores ciudadanos. De esta manera, el proceso de paz resulta beneficioso para el país, porque puede dar lugar, además de la cesación del conflicto y reformas políticas, a la apertura de nuevas fuentes de trabajo y empleo. 
En ese contexto, el emprendimiento entra a jugar un papel de importancia en los ámbitos social y económico, nacional y local, tanto para la población civil como para las personas que asumen el proceso de reinserción e igualmente para las víctimas del conflicto armado en Colombia. En este sentido, el interés del estudio es saber ¿cómo el emprendimiento puede constituirse en alternativa de reinserción productiva para las personas excombatientes y víctimas en el postconflicto?

En cuanto a emprendimiento, enmarcado en un ecosistema emprendedor para referirse a una correlación de fuerzas sociales en donde, si bien el emprendedor puede ser protagonista, de igual manera hace parte de un sistema productivo, solidario e inclusivo, constituido por un conjunto de prácticas y conceptos que contribuyen al logro de un determinado objetivo.

Bajo esta visión, el emprendimiento puede colaborar, en gran medida, en la reinserción efectiva a la sociedad de las personas que entregaron las armas. En realidad, el enfoque de derechos de los Acuerdos de paz, está orientado para que las medidas acordadas contribuyan a la materialización de los derechos constitucionales de los colombianos, es decir, lograr la incorporación a la sociedad de las personas que se han ubicado al margen de la ley, precisamente para que sean cubiertos por la legalidad $\mathrm{y}$, de igual manera, gocen de las garantías y derechos constitucionales.

La revisión sistemática se realizó para dar cuenta de los avances y tendencias en relación con la apertura de posibilidades productivas de poblaciones reincorporadas, victimas $y$, en general, de poblaciones vulnerables; más cuando en el momento se han agudizado los conflictos internos en algunos países. Profundizar en estos aspectos es importante en la medida en que se fortalece el cuerpo teórico referente a las opciones de productividad y emprendimiento, y amplía la visión desde una mirada interdisciplinar que puede favorecer la labor de diferentes profesionales en diferentes campos ocupacionales.

Así mismo, los resultados pueden ofrecer información valiosa a quienes se encuentran desarrollando herramientas tecnológicas y sociales para la comprensión de los conflictos humanos y para facilitar la comunicación por medios interpersonales y masivos. Así mismo, permite ampliar la visión de profesionales en el campo de la educación y el desarrollo humano, para cumplir con un papel social. 


\section{Metodología}

El proceso de revisión sistemática orientado a obtener una muestra final de documentos para el análisis, constó de tres partes: recopilación de datos, análisis de datos y síntesis. La estrategia de búsqueda consistió en la exploración de los términos generales en las bases de datos especializadas : Scopus, Scielo, Redalyc, Science Direct y Google Scholar.

Se utilizaron los términos de búsqueda Entrepreneurship and post-conflict, y, a la vez, se combinaron con sinónimos para lograr cubrir una mayor cantidad de documentos a evaluar.

En la Tabla 1 se muestra la cadena de búsqueda resultante del término, junto a sus sinónimos y correspondientes filtros.

\section{Tabla 1}

Cadena de búsqueda

\begin{tabular}{cl}
\hline Término principal & \multicolumn{1}{c}{ Cadena de búsqueda } \\
\hline \multirow{2}{*}{ Entrepreneurship and post- } & (TITLE-ABS-KEY (entrepreneurship) AND TITLE-ABS- \\
conflict & KEY ("civil war" OR"civil conflict" OR"postconflict" \\
& OR"post-conflict")) \\
\hline
\end{tabular}

Fuente: elaboración propia.

En la primera etapa, la recopilación de datos se inició con una búsqueda de la información contenida en las bases de datos mencionadas, a fin de centrar la atención en revistas examinadas por pares de forma análoga a otras revisiones efectuadas en el campo. La búsqueda se realizó en diciembre de 2019, con los criterios que se detallan en la Tabla 2. 


\section{Tabla 2}

Búsqueda en páginas Scopus, Scielo, Redalyc, Science Direct, Google Scholar y Dialnet

\begin{tabular}{|c|c|c|c|c|c|c|}
\hline Refinar & Scopus & Scielo & Redalyc & $\begin{array}{c}\text { Science } \\
\text { Direct }\end{array}$ & $\begin{array}{l}\text { Google } \\
\text { Scholar }\end{array}$ & Dialnet \\
\hline Cadena de búsqueda “(TITLE- & & & & & & \\
\hline ABS-KEY (entrepreneurship) & & & & & & \\
\hline $\begin{array}{l}\text { AND TITLE-ABS-KEY ("civil } \\
\text { war" OR"civil conflict" }\end{array}$ & 34.017 & 357 & 43.434 & 26712 & 27 & 252 \\
\hline $\begin{array}{l}\text { OR"postconflict" OR"post- } \\
\text { conflict"))" }\end{array}$ & & & & & & \\
\hline Tipo de acceso abierto & 2.886 & & & 4.037 & & \\
\hline Año (2009-2019) & 19.994 & 181 & 9813 & 3111 & 27 & 252 \\
\hline Nombre del autor (Dana, L.) & 124 & & & & & \\
\hline Nombre del autor Desai, S. & 2 & & & & & \\
\hline $\begin{array}{l}\text { Área temática (negocios, } \\
\text { administración y contabilidad) }\end{array}$ & 19.289 & 46 & 2.267 & & & \\
\hline Tipo de documento (Artículo) & 22.395 & 339 & & 664 & & 206 \\
\hline $\begin{array}{l}\text { Título de la fuente (Economía } \\
\text { de la pequeña empresa) }\end{array}$ & 601 & & & 6284 & & \\
\hline $\begin{array}{l}\text { Palabra clave (Espíritu } \\
\text { emprendedor) }\end{array}$ & 10.743 & 10 & & 252 & & 457 \\
\hline $\begin{array}{l}\text { Palabra clave (Emprendimiento } \\
\text { y postconflic to) }\end{array}$ & 16 & 1 & 43.671 & 177 & 769 & 114 \\
\hline $\begin{array}{l}\text { Afiliación (Universidad } \\
\text { Indiana) }\end{array}$ & 366 & & & & & \\
\hline $\begin{array}{l}\text { Patrocinador de financiación } \\
\text { (Fundación Nacional de } \\
\text { Ciencias) }\end{array}$ & 176 & & & & & \\
\hline $\begin{array}{l}\text { Patrocinador de financiación } \\
\text { (Dirección de Ciencias }\end{array}$ & & & & & & \\
\hline $\begin{array}{l}\text { Sociales, del Comportamiento y } \\
\text { Económicas (SBE)) }\end{array}$ & 1 & & & & & \\
\hline $\begin{array}{l}\text { País/territorio (Estados Unidos, } \\
\text { Colombia, México, España) }\end{array}$ & 9.298 & 210 & 26.402 & & & \\
\hline Tipo de fuente (Revistas) & 25.474 & & & & & \\
\hline Idioma inglés & 32.779 & 281 & 698 & & & \\
\hline Idioma español & 492 & 46 & 42.152 & & & \\
\hline Artículos pertinentes & 10 & 1 & 2 & 0 & 17 & 20 \\
\hline Total artículos a analizar & & & & & & 50 \\
\hline
\end{tabular}

Fuente: elaboración propia. 
El proceso de selección de estudios se realizó en cinco momentos (debido a que se consideró seis bases de datos especializadas), y la consulta se realizó en diversas fechas. Para cada momento, se tuvieron en cuenta tres fases:

Fase 1: Eliminación de artículos duplicados.

Fase 2: Eliminación de artículos no descargables.

Fase 3: Aplicación de criterios de inclusión y exclusión.

De los 50 artículos que cumplieron con los criterios de inclusión, para efectos del presente artículo, se tuvieron en cuenta únicamente 20 .

\section{Resultados}

Para la selección de los 20 artículos, se tuvo en cuenta el continente al cual pertenecen (véase Tabla 3); así, el mayor porcentaje se encontró que está en América, donde, a su vez, la mayoría de artículo s analizados son de Colombia, seguramente por su situación actual de conflicto y el prolongado tiempo que lleva la confrontación.

\section{Tabla 3}

Porcentaje de artículos por continente

\begin{tabular}{ccccc}
\hline América & Africa & Asia & Europa & Mundial \\
\hline $80 \%$ & $6 \%$ & $6 \%$ & $2 \%$ & $6 \%$ \\
\hline
\end{tabular}

Fuente: elaboración propia.

De igual manera, se consideraron los enfoques de investigación que se aplicaron en los documentos revisados, donde después de identificar que América obtiene el mayor porcentaje, el estudio se centra en Colombia específicamente, como se muestra en la Tabla 4.

\section{Tabla 4}

Enfoques de investigación

\begin{tabular}{cccc}
\hline Cualitativo & Cuantitativo & Mixto & Revisión documental \\
\hline $24 \%$ & $26 \%$ & $16 \%$ & $34 \%$ \\
\hline
\end{tabular}

Fuente: elaboración propia. 
Como se puede observar, en los artículos examinados predomina la revisión documental, seguida del enfoque cuantitativo, que resulta secuencial y probatorio. El orden que sigue es riguroso, aunque, desde luego, se puede redefinir alguna fase. Este parte de una idea que va acotándose y, una vez delimitada, se derivan objetivos y preguntas de investigación, se revisa la literatura y se construye un marco o una perspectiva teórica. De las preguntas se establecen hipótesis, se determinan variables y se traza un plan para probarlas -diseño-. Las variables se miden en un determinado contexto, con métodos estadísticos se analizan las mediciones obtenidas y se extrae una serie de conclusiones (Hernández et al., 2014).

En tercer lugar, se encontró que los artículos utilizan el enfoque cualitativo; en éste, en lugar de que la claridad sobre las preguntas de investigación e hipótesis precedan a la recolección y el análisis de los datos -como en los estudios cuantitativos-, permite plantear preguntas e hipótesis antes, durante o después de la recolección y el análisis de los datos. Con frecuencia, estas actividades sirven, primero, para descubrir cuáles son las preguntas de investigación más importantes $y$, después, para perfeccionarlas y responderlas. La acción e indagatoria se mueve de manera dinámica en ambos sentidos: entre los hechos y su interpretación, para resultar un proceso más bien circular, en el que la secuencia no siempre es la misma, pues varía con cada estudio (Hernández et al., 2014).

En cuarto lugar, los artículos utilizan el enfoque mixto, que es la combinación de los enfoques cuantitativo y cualitativo.

Por otra parte, se seleccionaron las diferentes estrategias de emprendimiento, presentes en los artículos, dirigidas a la población objeto de estudio, las cuales se presentan en la Tabla 5.

\section{Tabla 5}

Estrategias de emprendimiento para el conflicto y postconflicto

\begin{tabular}{lc}
\hline \multicolumn{1}{c}{ Estrategia } & Porcentaje \\
\hline Políticas públicas para emprendimiento & $20 \%$ \\
Apoyo de diversas entidades al emprendimiento & $20 \%$ \\
Impulsar diferentes tipos de emprendimiento & $14 \%$ \\
Impulsar el emprendimiento dirigido a diferentes tipos de poblaciones & $12 \%$
\end{tabular}


Generación de cooperativas, asociaciones y cadenas productivas para impulsar el emprendimiento

Intervención psicosocial para el emprendimiento

Técnicas para medir e incentivar el emprendimiento

$6 \%$

Educación empresarial

$6 \%$

Influencia de los valores culturales en el emprendimiento

$4 \%$

TOTAL $100 \%$

Fuente: elaboración propia.

Dentro de las estrategias de emprendimiento relacionadas con el conflicto y periodo de posconflic to, se destaca la de políticas públicas, como la Política de desarrollo agrario integral, orientada a promover la unión de las regiones y el desarrollo socio-económico y equitativo del país (Sanchez, 2016).

Otro es el Programa de Generación de Ingresos, como componente de la Política pública de atención al desplazamiento, por parte del Gobierno Nacional; éste establece el acompañamiento psicosocial hacia lo productivo, asesoría empresarial para la formulación y el desarrollo del plan de negocios y entrega de recursos no reembolsables para la financiación (de acuerdo a la firma de compromisos y control social).

Ejecutado por operadores a través de la formulación y ejecución de modalidades de atención, como: vinculación laboral, emprendimiento y fortalecimiento; así mismo, el plan complementa las acciones de atención integral para la población en situación de desplazamiento -al igual que: prevención, emergencias y retornos, atención humanitaria de emergencia, estabilización socioeconómica y atención territorial-, para contribuir con la estabilización socioeconómica de la población en situación de desplazamiento y como alternativa para las personas inscritas en el Registro Único de Población Desplazada (RUPD) antes del 31 de diciembre de 2005 (Herazo y Sellamén, 2010).

Para los excombatientes, el Estado colombiano brinda un apoyo legal y económico, que con base en la Ley 1429 de 2010 (Ley de Formalización y Generación de Empleo y, en particular, el Decreto 1391 de 2011), se reglamentan los subsidios o beneficios económicos para este fin particular. En dicha ley se destaca que el interés es apoyar a los pequeños emprendedores para que sus empresas se formalic en, 
o sea, queden legalmente constituidas ${ }^{1}$. El monto de capital semilla que se brinda, de acuerdo con el Decreto 128 de 2003, es de máximo ocho millones de pesos. Los futuros microempresarios pueden invertir ese dinero en emprendimiento, si es el caso de nuevo negocio, o en fortalecimiento para aquellos que ya lleven un año con su negocio.

En cuanto a la estrategia de apoyo de diferentes entidades al emprendimiento, es posible la optimización de resultados a través de la formulación de proyectos de emprendimiento por parte de mujeres víctimas del conflicto armado, propendiendo por su reparación, con un rol activo en ello; verbigracia, las convocatorias realizadas por el Banco de Desarrollo Empresarial (Bancoldex) que, actualmente, busca asignar recursos de cofinanciación no reembolsables a propuestas cuya finalidad sea crear modelos de microfranquicias para población víctima del desplazamiento forzado en Colombia, que permita el crecimiento de sus micro, pequeñas y medianas empresas (MIPYME) y la creación de unidades de negocio formales que garanticen la generación de ingresos sostenibles (Castillo y Picón, 2018).

Las instituciones microfinancieras (IMF) en Colombia, que tradicionalmente han sido ejemplo de inclusión financiera, pueden contribuir con los esfuerzos estatales para disminuir los efectos del conflic to armado. Su participación frente a un grupo especial de personas interesadas en reintegrarse a la sociedad, las convoca a identificar los retos que surgen de esta labor y a diseñar productos financieros y no financieros, sin comprometer su patrimonio, acordes con el perfil de los sujetos interesados en reemplazar su actividad como partícipes del conflicto armado, por actividades de emprendimiento empresarial. Se defiende la tesis que las IMF, no obstante, su carácter privado, pueden apoyar las políticas estatales para el posconflicto, mediante una ampliación de la cobertura en la prestación de los servicios microfinancieros en las zonas urbanas y en el sector rural.

Este microfinanciamiento de corto plazo, se otorga a personas vulnerables económicamente; el monto del crédito es relativamente bajo, debido a que se otorga sin garantías reales, como un crédito de confianza, a una tasa de interés superior a la que cobra la banca comercial, lo que permite cubrir los

\footnotetext{
${ }^{1}$ Durante los dos primeros años los exime de impuesto a la renta, el tercer año pagan un $25 \%$, el cuarto un $50 \%$, el quinto un 75\%, y es sólo a partir del sexto año que pagan la totalidad del impuesto correspondiente a renta. En igual medida, el pago de impuesto de industria y comercio, los pagos de parafiscales y otras contribuciones de nómina se hacen progresivas hasta el sexto año.
} 
riesgos en que se incurre; los recursos prestados se pagan en periodos cortos, con la ventaja de que los beneficiarios reciben de la IMF asesoría técnica especializada, que incluye visitas para verificar el estado de la actividad empresarial, analizar la operación crediticia y corroborar que el cliente tenga la capacidad y voluntad de pago necesaria para responder por la obligación (Grau-Prada, 2017).

En igual sentido, los propietarios de las empresas pueden comprometerse con la sociedad en el marco del conflicto. De la población de empresarios, la mayoría considera que debe esperar mayores resultados de las mesas de negociación, al considerar que el Gobierno debe implementar políticas claras, que faciliten al empresario la comprensión de cómo ejecutar las contrataciones laborales y, así, tomar las decisiones que les permita establecer las estrategias convenientes para contribuir con la empleabilidad en el posconflicto (García et al, 2018).

Otra de las formas de crear oportunidades desde las organizaciones empresariales para los desmovilizados y afectados por el conflicto, es a través de una mejor educación y capacitación. El sector privado puede contribuir al tener buenas prácticas y estrategias de responsabilidad social empresarial (Buitrago et al., 2015).

La ausencia de capacitación trae desventajas de tipo económico, como la baja productividad, menor calidad, deficiencia en la atención al cliente, desperdicio en materia prima e infrautilización de máquinas; así como comportamientos sociales como el ausentismo, la impuntualidad, abandono del puesto de trabajo, accidentes, bajas por invalidez e, incluso, muertes (Garrido-Hurtado, y ValderramaCardona, 2016).

La empresa norteamericana Coca-Cola Femsa, desde 2007 ha participado en la reintegración de excombatientes, apoyando a la Agencia Colombiana para la Reintegración (ACR) con el Programa de Paz y Reconciliación (Guaqueta y Orsini, 2007), el cual ha beneficiado a más de 400 reintegrados, a través de cuatro iniciativas: i) contribuyendo tiempo; ii) proyectos productivos; iii) centros de aprendizaje comunitario, y $i v$ ) espacios de paz y reconciliación. Sin embargo, la multinacional es blanco de críticas por parte de sindicatos como Sinaltrainal, que la acusa de patrocinar la muerte de sindicalistas, negociar con paramilitares, entre otros cargos (Jiménez, 2014). 
Colombia cuenta con más de veinte años de experiencia en procesos de reintegración voluntaria y coordinada por el Estado. A ello se suma que para la etapa de peacebuilding y la reintegración económica, buena parte del empresariado ha manifestado su apoyo, al ofrecer puestos de trabajo o patrocinar proyectos productivos, además del respaldo de la comunidad internacional para el periodo de posconflicto en Colombia (Cepeda, 2016).

Las propuestas del sector productivo implican la experiencia, formación y capacitación de los exmilitantes en sectores agrarios y urbanos como proyectos agropecuarios de impacto en el mercado o se fomente el emprendimiento para la generación de autoempleo entre jóvenes (Delgado et al., 2018).

Para ciertas empresas, los emprendimientos pueden llevar a capacitaciones conjuntas de población víctima, desplazados y reinsertados, lo que puede abrir el camino para generar espacios de integración y reconciliación; igualmente, generar alianzas para vincular a campesinos y víctimas como parte de su cadena de proveedores, eliminando intermediarios, lo cual representa una disminución en la estructura de costos para las empresas, y le permite tener control sobre su cadena de producción (Mariño-Arévalo, A. y Valencia-Toro, 2015).

En cuanto a las Instituciones de Educación Superior, éstas deberán gestionar retos en materia de políticas, programas y proyectos sociales, orientados al logro de tres objetivos: reconstrucción social, reconciliación humana y gestión de los conflictos para su resolución. Si bien es cierto que cada universidad es autónoma en la definición de políticas, se debe tener en cuenta el establecimiento de currículos a la luz de la responsabilidad social, interrelación entre las funciones sustantivas, medio ambiente, equidad educativa y de género, derechos humanos, producción del conocimiento, relación con egresados, vínculo con el sector empresarial, gobierno y demás grupos de interés, entre éstos el de los reinsertados.

En el caso particular de la Universidad Autónoma de Bucaramanga, esta ha tenido iniciativas como la de educación para la diversidad, con asignaturas enfocadas a lo socio humanístico y es, precisamente, en este espacio donde la institución brinda la oportunidad de hablar sobre el conflicto armado, 
posconflicto, ciudadanía, expresión cultural, como dirimir conflictos, emprendimiento y creatividad (Delgado et al., 2018).

Otra estrategia mencionada, es la relacionada con la educación empresarial como insumo para el emprendimiento en situaciones de conflicto y postconflicto. Para los excombatientes, el Estado colombiano ofrece apoyo de carácter técnico; las empresas que tienen convenios con la Agencia Colombiana para la Reincorporación (ACR), brindan capacitación y asesoría a los emprendedores; así, se creó el programa Aportando Tiempo, en el que las empresas delegan profesionales en temas de administración y gerencia para que los capaciten.

La educación empresarial tiene un efecto transformador en el comportamiento de las personas en un contexto de conflicto y, por lo tanto, altera sus circunstancias económicas de manera que reduzca la tendencia a apoyar el conflicto, con cambios en las actitudes y el pensamiento sobre el trabajo por cuenta propia, con la toma de conciencia sobre la naturaleza multidisciplinar del emprendimiento.

Por otra parte, la constitución de cooperativas también se ha convertido en una estrategia para impulsar el emprendimiento en contextos de conflicto y posconflicto, ya que se dispone de recursos para que los excombatientes generen cooperativas y pequeñas microempresas como fuentes de empleo y de ingresos; aquellos que están organizados en gremios obtienen mayores beneficios. Por la dinámic a gubernamental de dar soluciones a grupos pequeños, se ha fomentado la proliferación de pequeñas asociaciones gremiales, lo que a largo plazo, en lugar de fortalecer la lucha, les ha debilitado como colectivo (Chamorro, 2015).

El papel del cooperativismo es un reto que tiene el Gobierno Nacional, el Ministerio de Trabajo y la Unidad Administrativa Especial de Organizaciones Solidarias. Los gremios, bajo la inclusión social, económica y cultural, desarrollan proyectos productivos de generación de ingresos y trabajo digno, para suplir al Estado en facilitar el acceso a la educación, la construcción y reparación de tejido social en zonas amenazadas por el conflicto, como las cooperativas lecheras en el Departamento de Antioquia, las cooperativas cafeteras en el Magdalena medio del Plan de Desarrollo y Paz, o la convulsionada región del Catatumbo en el Departamento de Norte de Santander, para citar algunos ejemplos (Herrera, 2017). 
La economía social y solidaria puede garantizar una paz social con justicia y equidad, acompañada por programas, políticas y estrategias gubernamentales para fomentar y fortalecer las iniciativas de asociación, las cuales pueden resultar débiles en sus lazos de confianza y solidaridad por falta de conciliación de los intereses y conflictos, las prácticas desleales del capitalismo, el bajo nivel de solidaridad y asociatividad y el individualismo, que igual debilitan la convivencia y producción colectiva (Serna y Rodríguez, 2015). No obstante, en cuanto al Estado "es también su deber solucionar problemas como la falta de oportunidades, la pobreza y la violencia, que hacen que una de las pocas formas de salir adelante, en territorios rurales especialmente, sean los modelos asociativos" (Callejas, 2017, p. 189).

El conflicto ha afectado a diferentes sectores de población, con quienes, en el periodo de postconflic to, también se ha impulsado el emprendimiento.

Emprendimiento en el sector juvenil. Bajo la Ley 1014 de 2006 o Ley de Fomento a la Cultura del Emprendimiento.

La Ley 1780 de 2016 o también conocida como Ley Pro-Joven, se promulgó con el objetivo de generar empleo para los jóvenes con edades entre 18 y 28 años, mediante el diseño y ejecución de políticas de empleo, emprendimiento y la creación de nuevas empresas jóvenes, junto con la promoción de mecanismos que impacten positivamente en la vinculación laboral de la población joven en Colombia.

La ley brinda garantías como alivios tributarios y no tendrán que realizar aportes a Cajas de Compensación Familiar por tales trabajadores durante el primer año de vinculación, para las empresas que contrate jóvenes y promuevan su acercamiento al sector productivo mediante la creación de pequeñas empresas, en especial para la población rural, minorías étnicas y jóvenes que se han acogido al Acuerdo de paz.

De acuerdo a Hoyos y Cabezas (2016, p. 9): "La formulación de este tipo de iniciativas, permite el fomento del emprendimiento en jóvenes, como una forma de superar la barrera del desempleo juvenil y las trabas de acceso al mercado laboral, tarea ardua en el contexto Colombiano". 
Por lo tanto, ofrecer oportunidades de empleo a los jóvenes o de generación de empresa, con apoyo y patrocinio del sector empresarial, evita que tengan que recurrir a grupos delincuenciales o al margen de la ley (Avendaño et al, 2017). Así mismo, la familia desempeña una función importante, tanto en el desarrollo de las aspiraciones de emprendimiento como en el apoyo económico y moral. Además, la recurrencia de los emprendedores jóvenes a las redes profesionales para adquirir conocimientos y apoyo material, se muestra de suma importancia, por la falta de programas para realizar estudios específicos sobre emprendimiento, lo cual los emprendedores jóvenes tienen que aprender mediante la práctica y reflexión sobre sus propias experiencias para continuar con el desarrollo de su negocio (Minialai et al, 2018).

Aunque los estudios de caso realizados, muestran que los jóvenes son capaces de iniciar y desarrollar sus empresas y tener éxito, también deben afrontar numerosos problemas, relacionados con la dependencia de su contexto social y económico, lo cual los hace vulnerables. En Colombia, la mayoría de jóvenes asumen el emprendimiento por necesidad ante la carencia de empleo; en estos casos, la travesía que deben recorrer supone un trayecto de supervivencia y de dignidad, de posición social, para conseguir autonomía en un entorno difícil (Minialai et al., 2018).

Emprendimiento en poblaciones indígenas. La gran variedad de comunidades indígenas y su riqueza cultural en Colombia, requiere un análisis de los factores que inciden en el perfil que se quiere identificar y que busca la promoción del emprendimiento y la asociatividad. La idea de emprendimiento en la población indígena a nivel nacional, podría ser acogida al momento de proyectar programas de desarrollo para su sostenimiento y progreso comunitario. Esto indica que el perfil del emprendedor indígena del país, se puede caracterizar por conservar rasgos tradicionales; a partir de esta característica, pueden generarse actividades productivas a corto, mediano y largo plazo. Para que estos procesos logren el impacto deseado, el emprendimiento y la asociatividad deben ser estimulados para que el individuo o la comunidad, se involucren de manera voluntaria y contemplando todas las variables culturales, tradicionales y sociales propias de cada población (Socarrás, 2004, citado en Pitre- Redondo et al., 2017, p. 237).

Por lo anterior, para desarrollar programas de fomento del emprendimiento, asociatividad y productividad en comunidades indígenas, éstos se deben enfocar en su sabiduría y tradición, tener en 
cuenta escuelas de trasmisión del conocimiento propio, donde los indígenas mayores difundan su sabiduría entre los jóvenes y niños, para que no desconozcan la historia como principal patrimonio y, con esta base, incentivar las actividades productivas. En la nueva etapa que está iniciando Colombia, indígenas desplazados regresarán a sus territorios de origen y el emprendimiento puede contribuir con la reconstrucción de estos escenarios (Pitre-Redondo et al., 2017).

Algunos tipos de emprendimiento que se pueden promover con personas o grupos reincorporados después del conflicto y en el periodo de postconflicto, pueden ser:

Emprendimiento turístico. A nivel de Colombia, se realizó una investigación donde se extractó las debilidades, amenazas, fortalezas y oportunidades del Departamento de Chocó, y se propuso una serie de acciones para mitigar los impactos en el territorio e identificar los posibles escenarios en que se puede dar la inclusión laboral de los desmovilizados (Crespi-Vallbona et al., 2019). Una vez identificados los atractivos naturales del área de intervención, se creó un producto turístico sostenible, el cual implicó el diseño de senderos para excursiones -donde los desmovilizados fueron escogidos como guías, ya que conocen el territorio-, y el diseño de rutas turísticas al río Atrato, puesto que conecta comunidades en sus riveras, con lo cual se incentivará el turismo comunitario en la zona.

Además, se investiga los potenciales y las limitaciones del emprendimiento social para lograr el turismo inclusivo, aunque en Colombia, esto tiene limitaciones. Conjuntamente, los beneficios sociales continuos dependen del éxito comercial de las empresas, en un sector altamente volátil y, para el caso de la región del litoral pacífico colombiano, en un estado de violencia casi continua; incluso, negocios bien administrados que no resultan completamente seguros. Existen empresas turísticas convencionales - una empresa de turismo, un hotel, un restaurante, una producción de objetos de recuerdo y ventas minoristas-, que han integrado una misión social en su modelo de negocio de diferentes maneras (Biddulph, 2018).

Emprendimiento rural. En lo relativo a la reinserción y la reintegración, las personas amnistiadas fueron beneficiadas con programas de entrega de tierras, apoyo para vivienda rural, créditos y algunos compromisos de atención social. Así, éstos tendieron a vincularse de nuevo al campo, en actividades 
productivas, en lugares distintos a aquellos donde hicieron parte de las guerrillas, e, igual, sobresalieron con frecuencia por el liderazgo social.

En lo referente a las microempresas, a pesar de las muchas experiencias negativas, es necesario reconocer la respuesta altamente positiva de varios excombatientes, quienes supieron valorar el significado de las diversas ayudas y, no obstante, los tropiezos y dificultades, han salido airosos en los proyectos que emprendieron. En cuanto a lo económico, con el apoyo del programa de reinserción, surgieron proyectos empresariales colectivos e individuales, rurales y urbanos, los cuales, sin embargo, están siendo afectados por la ausencia de una estrategia acertada, ya que se tiende al predominio de un enfoque hacia la individualización, también existen falencias en la asesoría, el seguimiento y notorias dificultades en el contexto macroeconómico (Franco, citado en Villarraga, 2013, p. 123).

Por ello, la investigación y formación en el ámbito empresarial, busca consolidarse a partir de la apropiación de la cultura emprendedora por parte de las comunidades en general y de manera específica en las rurales (Tovar et al., 2017).

Emprendimiento destructivo. El concepto de emprendimiento destructivo, aunque se menciona desde hace más de 25 años, continúa siendo una especie de caja negra en la literatura sobre emprendimiento y economía; sin embargo, promete colaborar con los enfoques económicos de la seguridad en las regiones en conflicto. En esta investigación, se aporta una idea de cómo los incentivos subyacentes al emprendimiento destructivo, pueden ser útiles para el diseño de los programas de desarme, desmovilización y reintegración (DDR) (Douhan y Henrekson, 2008; Desai, et al., citados en Desai, 2016, p. 241).

Los programas de desarrollo alternativo buscan alejar a los agricultores de la producción de productos básicos de alto valor como la coca y la amapola, y orientarlos a otros como el café y las nueces, pero han tenido resultados mixtos, en gran parte sin éxito. También existe el problema de la sostenibilidad y la gestión del programa, porque varios programas DDR han fallado cuando, después de la transic ión de los actores internacionales a las autoridades locales, las compensaciones fueron mal administradas, reducidas o detenidas (Desai, 2016). 


\section{Conclusiones}

El emprendimiento está relacionado, no solamente con la creación de empresas como comúnmente se lo asocia sino, también, con el crecimiento de las personas y de las comunidades, al involucrase en un entorno de educación y cultura, como una actitud con base en el saber y en las prácticas emprendedoras.

El emprendimiento es una alternativa para la solución de las problemáticas reales que enfrenta la población en proceso de reinserción, lo mismo que las víctimas del conflicto, por cuanto no sólo beneficia a una persona en particular, sino que propicia el desarrollo de toda la comunidad, mejora la calidad de vida de las familias y promueve la innovación para agregar valor a los productos.

Las políticas públicas para incentivar el emprendimiento, al no traducirse en planes de acción concretos, dificultan la articulación de ellos a cada uno de los programas estatales, reduciendo su capacidad para implementar lo establecido en las políticas, y notándose, además, un débil acompañamiento institucional a nivel nacional y local.

Las instituciones de educación, las empresas y el Estado se han constituido en actores claves para apoyar al emprendimiento en situaciones de conflicto y en el periodo de postconflicto, al unir sus fortalezas y proponer planes factibles de implementación en beneficio de la población víctima de la situación actual.

El papel de la educación en los procesos de emprendimiento se ha constituido como fundamental, al acompañar en la formulación de proyectos con sus objetivos, metas, estrategias y acciones que vinculan a este sector con las problemáticas de la sociedad y, en particular, con los procesos de cesación del conflicto armado y posterior periodo de paz.

Si bien el emprendimiento constituye una estrategia válida de inclusión y productividad para las personas en proceso de reinserción a la sociedad, esto necesita de un proceso educativo para la apropiación de conceptos y prácticas para la creación de empresas y proyectos productivos. 
En el momento se considera que los excombatientes cuentan con recursos humanos y económicos para generar cooperativas, asociaciones y cadenas productivas como fuente de generación de empleo y de ingresos; la organización en gremios permite obtener mayores beneficios. Es de destacar que el emprendimiento va de la mano de la asociatividad, la inclusión social y la economía, como estrategias de reinserción, reconciliación y fortalecimiento de la paz.

Dentro del emprendimiento para víctimas y excombatientes, se plantean diferentes tipos al tener en cuenta las potencialidades de las regiones donde se encuentran, por ejemplo, el emprendimiento turístico, rural, cultural, social y otros.

Los inmigrantes y personas en condición de desplazamiento en Colombia y en otros países, tienden a tener tasas más altas de autoempleo que los nativos. La política de emprendimiento y la de pequeñas y medianas empresas (PYME) buscan fomentar la formación en emprendimiento como trabajo por cuenta propia o propiedad de pequeñas empresas.

En el sentido en que la investigación indagó sobre emprendimiento en otros países que también han cruzado por periodos de conflicto armado interno, como denominador común se encontró que para las personas excombatientes y víctimas, es importante la educación, el apoyo familiar en lo económico y moral, las redes profesionales a las que recurren los jóvenes para adquirir conocimientos y las políticas públicas en el desarrollo de las aspiraciones de emprendimiento.

\section{Referencias}

(1) Avendaño, M., Mondragón, S. y Caballero, M. (2017). Plan prospectivo y estratégico sobre la reinserción social a partir del postconflicto al año 2023. Documentos de Trabajo ECACEN, 1(1), 1-11. http//hemeroteca.unad.edu.co/index.php/working/article/view/2560/2672

(2) Biddulph, R. (2018). Social enterprise and inclusive tourism. Five cases in Siem Reap, Cambodia. Tourism Geographies, 20(4), 610-629. https://doi.org/10.1080/14616688.2017.1417471

(3) Buitrago, N., Bustamante, A., Castañeda, S. y Ramírez, S. (2015). Retos de las organizaciones privadas en el postconflicto colombiano. Ensayos: Revista De Estudiantes De Administración De 
Empresas, (8), 228-239. https://revistas.unal.edu.co/index.php/ensayos/article/view/56333

(4) Callejas, C. (2017). Factores de éxito de la Asociación de Tecnólogos y Productores de Bilbao, una empresa asociativa en el posconflicto. Equidad y Desarrollo, (27), 169-194. https://doi.org/10.19052/ed.3985

(5) Castillo, S. y Picón, M. (2018). La mujer como sujeto partícipe de reparación en el contexto del posconflicto en Colombia. Gênero \& Direito, 6(3), 72-86. https://doi.org/10.22478/ufpb.21797137.2017v6n3.35309

(6) Cepeda, J. (2016). El posacuerdo en Colombia y los nuevos retos de la seguridad. Cuadernos de Estrategia, 181(1), 195-224. https://dialnet.unirioja.es/servlet/articulo?codigo=5673544

(7) Chamorro, S. (2015). Acercamiento al proceso de Desarme, Desmovilización y Reinserción (DDR) en Nicaragua después de 28 años de la firma de los acuerdos de Esquipulas II. Cultura de $P a z, 21(65), 15-27$. https://doi.org/10.5377/cultura.v21 i65.1970

(8) Crespi-Vallbona, M., Galeas, S. y López, M. (2019). Desarrollo turístico inclusivo socialmente. El caso de los desmovilizados en la región del Chocó, Colombia. Cuadernos Geográficos, 58(1), 157-179. https://doi.org/10.30827/cuad geo.v58i1.6759

(9) Delgado, M., Forero, V., Martínez, C., Sanabria, P., Serrano, W. y Tello, A. (2018). La reintegración social en el posconflicto, una mirada desde Bucaramanga, Santander: Convivencia y nivel de preparación [Tesis de pregrado, Universidad Autónoma de Bucaramanga, Colombia]. https://www.unab.edu.co/sites/default/files/archivos/publicacion_noticias/LA\%20REINTEGRA CI\%C3\%93N\%20SOCIAL\%20EN\%20EL\%20POSCONFLICTO\%2C\%20UNA\%20MIRADA \%20DESDE\%20BUCARAMANGA\%2C\%20SANTANDER.pdf

(10)Desai, S. (2016). Destructive entrepreneurship and the security context: Program design considerations for disarmament, demobilization and reintegration (DDR) and counterinsurge ncy. Journal of Entrepreneurship and Public Policy, 5(2) 240-250. https://doi.org/10.1108/JEPP-032016-0009

(11) García, J., Duran, S., Parra, M. y Martínez, H. (2018). Inserción, integración y equidad en el ámbito laboral: Escenario empresarial posconflicto en Colombia. Revista de Ciencias Sociales, 24(3), 36-49. https://dialnet.unirioja.es/servlet/articulo?codigo=7025046

(12) Garrido-Hurtado, D. y Valderrama-Cardona, E. (2016). Identificación de las ideas de negocio para la creación de empresa en la población desplazada de Florencia, Colombia. Revista de $\begin{array}{llll}\text { Investigación, } \quad \text { Desarrollo } & \text { Innovación, } & \text { 3(1), } & \text { 37-48. }\end{array}$ 
https://doi.org/10.19053/20278306.v7.n1.2016.5634

(13) Grau-Prada, J. (2017). El papel de las instituciones microfinancieras en el posconflicto en Colombia. Díkaion, 26(2), 283-312. https://doi.org/10.5294/dika.2017.26.2.4

(14)Guaquetá, A. \& Orsini, Y. (2007). Business and reintegration: Cases, experiences and lessons. Fundación Ideas para la Paz.

(15) Herazo, G. y Sellamén, A. (2010). El programa de generación de ingresos y el desplazamie nto forzado. Criterio $\quad$ Libre, $\quad$ 8(13), 129-170. http:/www.unilibre.edu.co/CriterioLibre/image s/revistas/13/art04.pdf

(16) Hernández, R., Fernández, C. y Baptista, M. (2014). Metodología de la investigación (6. ${ }^{\text {eed.). }}$ McGraw Hill, Editorial.

(17) Herrera, A. (2017). Las políticas públicas como alternativa estructural para el desarrollo rural solidario y posconflicto colombiano. Revista de la Universidad de La Salle, (74), 72-100. https://dialnet. unirioja.es/servlet/articulo?codigo $=6636410$

(18) Hoyos, S. y Cabezas, F. (2016). Análisis de política de empleo y emprendimiento juvenil en un escenario de posconflicto. Repositorio Institucional Sergio Arboleda. https://repository.usergioarboleda.ed u.co/bitstream/handle/11232/746/An\%C3\% A1 lisis\%20de $\% 201$ \% $\% 20$ pol\%C3\%ADtica\%20de\%20empleo.pdf?sequence $=1$

(19) Jiménez, G. (2014). Multinacionales y responsabilidad social empresarial en la construcción de paz en Colombia. Cuadernos de Administracion, 27(48), 67-96. http://www.scielo.org.co/pdf/cadm/v27 n48/v27n48a04.pdf

(20)Ley 1014 de 2006. (2006, 26 de enero). Congreso de la República de Colombia. Diario oficial No. 51.478. http://www.secretariasenado.gov.co/senado/basedoc/ley_1014_2006.html

(21)Ley 1429 de 2010. (2010, 29 de diciembre). Congreso de la República de Colombia. Diario oficial No. 51.478. http://www.secretariasenado.gov.co/senado/basedoc/ley_1429_2010.html

(22)Ley 1780 de 2016. (2016, 2 de mayo). Congreso de la República de Colombia. Diario oficial No. 49.861.

http:/es.presidencia.gov.co/normativa/normativa/LEY\%201780\%20DEL\%2002\%20DE\%20M AYO\%20DE\%202016.pdf

(23) Mariño-Arévalo, A. y Valencia-Toro, M. (2015). "Participación de la gran empresa en la política pública de atención a las víctimas del conflicto armado en Colombia". Cuadernos de Administración, 28(50), 159-185. https://doi.org/10.11144/javeriana.cao28-50.pgep 
(24)Minialai, C., Bossenbroek, L. y Ksikes, D. (2018). ¿Es el emprendimiento una salida para la juventud marroquî? Revista cidob d'afers internacionals, 8(118), 35-56. https://doi.org/doi.org/10.24241/rcai.2018.118.1.35

(25)Pitre-Redondo, R., Cardona-Arbeláez, D. y Hernández-Palma, H. (2017). Proyección del emprendimiento indígena como mecanismo de competitividad en el postconflicto colombiano. Revista de Investigación, Desarrollo e Innovación, 7(2), 231-240. https://doi.org/10.19053/20278306.v7.n2.2017.6068

(26) Presidencia de la República de Colombia, Oficina del Alto Comisionado para la Paz. (2016). Acuerdo final para la terminación del conflicto y la construcción de una paz estable y duradera. https://www.cancilleria.gov.co/sites/default/files/Fotos2016/12.11_1.2016nuevoacuerdofinal.pd $\mathrm{f}$

(27) Sanchez, S. (2016). Sobre las leyes para la restauración de víctimas y la reinserción de rebeldes armados. Revista Jurídica Primera Instancia, 4(7), 143-167. http:/www.primerainstancia.com.mx/wp-content/uploads/2017/04/SOBRE-LAS-LEYES-

PARA-LA-RESTAURACI\%C3\%93N-DE-V\%C3\%8DCTIMAS-Y-LAREINSERCI\%C3\%93N-DE-REBELDES-ARMADOS.-Sandra-S\%C3\%A1 nchez-Velandia..pdf

(28) Serna, H. y Rodríguez, M. (2015). El sector solidario como alternativa para el desarrollo social e inclusivo en el postconflicto colombiano. Cooperativismo \& Desarrollo, 23(107), 53-78. https://doi.org/10.16925/co.v23i107.1250

(29)Tovar, M., Babativa, D. y Reyes, G. (2017). Cultura emprendedora: estrategia para la productividad y competitividad de la nueva ruralidad en el posconflicto. La Restauración Social en el Posconflicto Como Herramienta para la Construcción de Paz, 1(1), 115-126. https://eresearch.areandina.edu.co/discover/display/n30277

(30) Villarraga, A. (2013). Experiencias históricas recientes de reintegración de excombatientes en Colombia. Colombia Internacional, (77), $107-140$. https://doi.org//10.7440/colombiaint77.2013.05

Cómo citar este artículo: Solarte, C., Solarte, M. y Rivera, G. (2021). Papel del emprendimiento en el conflicto y postconflicto: una revisión sistemática de literatura. Tendencias, 22(1), 204-225. https://doi.org/10.22267/rtend.212201.161 